Schweblin und ihr Roman Hundert Augen, in dem ein Panda-Roboter uns mit der Offenlegung unserer persönlichen Geheimnisse erpresst, und Valeria Lusellis Archiv der verlorenen Kinder. Die Essay-Chronik erzählt, ausgehend von ihrer Erfahrung als Dolmetscherin für Migrantenkinder, aus deren Perspektive und in Worten voller Misstrauen von ihrem Weg in die USA, nachdem sie den Massengräbern nahe der Grenze entkommen sind. Die Kinder wagen daraufhin das Abenteuer, von einem Land abgelehnt zu werden, das Waffen nach Lateinamerika liefert.

\title{
Was sollen wir tun?
}

Das Wissen, was durch die Erfassung und Ordnung großer elektronischer Datensätze wie auch durch deren Kritik entstanden ist, weist nach wie vor Lücken auf. Dieser Umstand wird noch durch die Annahme verschärft, dass unterschiedliche Kulturen und Formen der sozialen Organisation pauschal zusammengefügt werden können. Uns Bürgern bleiben das Essay und die Montage als Strategien des Gegenangriffs und zur Aufdeckung der Probleme. Es ist erforderlich, eine vorläufige Staatsbürgerschaft $\mathrm{zu}$ entwickeln und sie in flexibler Zusammenarbeit auszuprobieren.

Die Komplexität der Transformationen und die Ausdehnung der globalisierten Interaktionen haben die eurozentrischen oder euroamerikanischen Konzepte, die das Zusammenleben in erster Linie im Rahmen der Modernität und des Nationalstaats vorsahen, unzulänglich gemacht. Es gibt keine globalen Organismen, die ausreichende Politiken liefern, weder für das Ausmaß der Konflikte noch für den interkulturellen Konsens über das Gemeinschaftliche, das Öffentliche und das Private, die Bürgerrechte, Gerechtigkeit oder die Arten, sie auszuüben. Innerhalb einer jeden Nation schränkt die Diskreditierung von Parteien, Gewerkschaften und anderen vermittelnden Institutionen den gemeinsamen sozial-regulierten Raum weiter ein, der zudem noch von autonomen Initiativen gemieden wird.

Diese zwischen den Bürgern und der öffentlichen Hand entzweiten Bereiche werden von messianischen Anführern neu aktiviert, indem sie klientilistische Anwerbungsstrategien verfolgen, bürgerliche und moralische Aufforderungen äußern, die sich auf der vorpolitischen Ebene bewegen und daher die unabhängige Organisation der Bürger behindern. Die religiösen Bewegungen, darunter die neoevangelikalen mit ihrer Propagierung von Schutz, Solidarität und einer ausgleichenden Geselligkeit, unterstützen oft neolibera- 
le Politiker mit teils improvisierten Karrieren (Bolsonaro ist der bekannteste, aber nicht der einzige Fall). Sie blockieren die eigentliche politische Partizipation und Kritik.

Die Rolle der Zuneigung und des Imaginären auf der kollektiven Ebene, die diese Grenzen verschieben, ist nicht zu unterschätzen. Aber letztendlich festigen sie die Abhängigkeit und den Verlust. Sie ordnen den öffentlichen Sinn des Konflikts anderen Formen unter, die auf die Zugehörigkeit und den Beitritt in eine Gruppe gerichtet sind, und sie untergraben individuelle Emanzipationsprojekte und schwächen selbstverwalteten Kollektive.

Die Enteignung der Gefühle wird auch von Unternehmen und Institutionen praktiziert. Zwei der Bewegungen empörter Bürger, die in diesem Buch analysiert wurden, wurden zu deren Opfer. Die "15M«-Demonstrationen in Madrid, die auf ihren Transparenten den Slogan "Otro mundo es posible« (dt. »Eine andere Welt ist möglich«) nutzten, sahen diesen später in Werbespots von Coca-Cola, die den Spruch übergenommen hatten. Ihre teilweise Entsprechung in Mexiko, nämlich die Bewegung »YoSoy132«, mit Ursprung an der Universidad Iberoamericana unter der Konfrontation des Präsidentschaftskandidaten der PRI und der darauffolgenden Verleumdung der Bewegungsmitglieder als Eindringlinge in die Institutionen, konterte dieses Manöver. Wie bereits einige Kapitel zuvor beschrieben, filmten 131 Studierende sich selbst und veröffentlichten ein Video, in dem sie ihren Status als Studierende bewiesen; die Legitimität und ihre Auswirkungen wurden kurz darauf von der Universidad Iberoamericana zweckentfremdet, indem sie städtische Werbetafeln der Institution mit dem übernommenen Hashtag \#YoSoyIbero (»Ich bin Ibero«) und mit einem jugendlichen Gesicht und Sätzen versahen, die von einer sozialen Innovation anhand der Kapazität »meiner Universität« sprachen.

Die Vermittlung über das Fernsehen und die soziodigitalen Netzwerke hat die Kommunikation ausgebaut und Wege geschaffen, die Partizipation möglich machen. Auch wenn sie manchmal erlauben, dass Zuschauer sich aktiv beteiligen, lassen sie wenig Raum für deren Partizipation als Bürger. Die jüngste Phase dieser Vermittlung über das Fernsehen und die Netzwerke wird mit der Verrechtlichung der Politik in Verbindung gebracht. In Argentinien, Brasilien, Kolumbien, Peru und in anderen Ländern übernehmen die Gerichte angesichts der andauernden Korruption die Verantwortung für die politischen Dispute in Bezug auf die Anschuldigungen gegen Parteiführer. Bevor es zum Urteil kommt und Beweise ausgewertet werden, kommt es zu einer »Anklage« in den Medien und im Netz, sowie zu einem media- 
len Prozess, der im Zusammenhang der öffentlichen Meinung verschiedene Verdächtigungen antreibt. Neben der Verschiebung von der politischen Polemik zu gerichtlichen Entscheidungen treten auch die Schwierigkeiten der Bürger in den Vordergrund, sich richtig und verlässlich zu informieren: Ihre Rolle wird insbesondere dadurch neutralisiert, dass das demokratische $\mathrm{Zu}$ sammenspiel durch gleichermaßen juristische, unternehmerische und parteiaffine Ebenen absorbiert wird. Die gelegentlichen Straßendemos sind das einzige Mittel, um sich dagegen zu behaupten.

Das gigantische Wissen der Algorithmen und ihre Fähigkeit zum Verflechten von Millionen von individuellen Verhaltensweisen erscheinen als die neue strukturgebende Macht. Aber die totalitäre Logik in ihrer Aneignung persönlicher Daten und die Unzulänglichkeit der algorithmischen Systeme bei der Erschaffung sozialer Gouvernementalität lassen die Bürger, Verbraucher und Nutzer außen vor, ohne dass wirksam dagegen vorgegangen wird.

Die Frage »Was sollen wir tun?« ordnet sich der Frage »Was können wir tun? « unter, wenn es den lateinamerikanischen Regierungen an politischen Konzepten und an Organismen mangelt, durch die post-digitale Schieflage kanalisiert werden kann. So wie sie (bis auf ein paar Ausnahmen) gleichgültig gegenüber den akademischen Erkenntnissen waren, die während des massiven Aufschwungs im Kommunikationssektor und der darauffolgenden Förderung von Freihandelsabkommen für eine öffentliche Medienpolitik und regionale Integration plädierten, handeln die Regierungen jetzt so, als hätten weder Handys, noch Tech-Giganten unsere Länder erreicht. Ausschließlich Verbraucher- oder Verbände für Informationsrecht sind an der Debatte beteiligt, die in Europa im April 2018 zur Verabschiedung der DatenschutzGrundverordnung (DGSVO) geführt hat, die für alle Unternehmen, Finanzkontrollen und den Handel mit persönlichen Informationen gilt. In den USA hat das Repräsentantenhaus Untersuchungen zu Angriffen auf den Wettbewerb und auf die Cyber-Sicherheit eingeleitet. In den Kampagnen vor den Wahlen Mitte 2019 wurden Vorschläge zur Zerschlagung von Firmen sowie für neue Regulierungen geäußert, die die zügige Begrenzung der Macht einzelner Unternehmen bewirken sollen. In Lateinamerika gibt es wissenschaftliche und journalistische Informationen die deutlich beweisen, dass Google seine eigenen Produkte bei der Suche favorisiert, und dass Facebook, Instagram und WhatsApp die soziodigitalen Netze monopolisieren. Amazon kontrolliert mit Handelsprivilegien den Online-Handel, aber die Gesetze ändern sich dahingehend nicht. Wie bisher weiterzumachen bedeutet, dass diese Missbräu- 
che zwischen Washington, Peking, Brüssel und Moskau geregelt werden also dort, wo Lateinamerikaner nur als unbedeutende Ausländer gelten.

Wir haben das demokratisierende Potenzial digitaler Ressourcen und die verschiedenen Gebräuche erkannt, die Jugendliche aus ihnen ziehen, um andere Wege zum Aufbau einer Gesellschaft zu ebnen. Des Weiteren haben wir einige kreative und gesellschaftlich-innovative Erfahrungen kennengelernt, sowie Projektaktionen, die bewusst von der Programmierung der Arbeit und der Kommunikation innerhalb des E-Kapitalismus abweichen. Trotzdem bleibt ihr emanzipatorischer Wert nicht ohne Zweifel, wenn wir ihn innerhalb der prekären Logik des sozioökonomischen Systems betrachten. Verbunden mit Jugendbewegungen die sich emanzipieren, vor allem unter Beteiligung junger Menschen mit einem hohen Bildungsniveau, münden das Nichtvorhandensein einer Regulierung seitens des Staates und dessen Zersetzung in vielen Fällen in das Entstehen illegaler und selbstzerstörerischer Netzwerke. Es bleibt daher die Frage: Was kann getan werden, um sicherzustellen, dass anfällige, kurzlebige Projekte zu einer Gesellschaft führen, die die jüngste Generation (sowie Rentner und Ausländer) schlussendlich nicht auf die hinteren Plätze verbannt und übergeht? Angesichts der Schwierigkeit, auf diese Frage eine Antwort zu finden, verweist man gerne auf Hacking, um die algorithmische Welt zu unterbrechen und uns in den Lücken einzurichten.

Ich füge eine weitere Aktionslinie hinzu: Unabhängige Vereinigungen der Zivilgesellschaft interpellieren die Staaten und internationalen Organisationen. Hier gibt es in Lateinamerika bereits einige Erfahrungen, unter denen die Equipos de Antropología Forense eine besondere Hervorhebung verdienen. Man könnte an dieser Stelle viele Unterfangen zur Erneuerung seitens der neuen Generation oder Verbände mit jugendlicher Beteiligung hinzufügen - Musikfestivals, Festivals zum unabhängigen Kino und Dokumentarfilmen mit breiter Rezeption (Ambulante in Mexiko, Baci in Buenos Aires etc.). Sie artikulieren innovative Initiativen mit öffentlichen und privaten Institutionen. Diese kulturellen und kommunikativen Aktivitäten stehen im Kontrast zur so genannten der Entfremdung der Jugendlichen gegenüber öffentlichen Institutionen. Kooperationsorganismen können einen neuen Sinn finden, sofern sie mit diesen Unterfangen verflochten werden und es schaffen, dass Jugendliche ihre Strukturen und Wege, Kultur zu machen und sie zu teilen, als interessant erachten. Durch die Fähigkeit, in die Richtung zu repräsentieren, in die sich die Gesellschaft bewegt, können die Integrationsorganismen mehr sein, als nur entfernte verstreute Platzhalter. 